\title{
W state generation and effect of cavity photons on the purification of dot-like single quantum well excitons
}

\author{
C.-M. Li ${ }^{1}$, Y.-N. Chen ${ }^{1}$, C.-W. Luo ${ }^{1}$, J.-Y. Hsieh ${ }^{2}$, D.-S. Chuu ${ }^{*}$ \\ ${ }^{1}$ Institute and Department of Electrophysics, National Chiao \\ Tung University, Hsinchu 30050, Taiwan. \\ ${ }^{2}$ Department of Mechanical Engineering, Ming Hsin University \\ of Science and Technology, Hsinchu 30401,Taiwan.
}

September 24, 2018

\begin{abstract}
A scheme of three-particle entanglement purification is presented in this work. The physical system undertaken for investigation is dot-like single quantum well excitons independently coupled through a single microcavity mode. The theoretical framework for the proposed scheme is based on the quantum jump approach for analyzing the progress of the trible-exciton entanglement as a series of conditional measurement has been taken on the cavity field state. We first investigate how cavity photon affects the purity of the double-exciton state and the purification efficiency in two-particle protocol. Then we extend the two-particle case and conclude that the three-exciton state can be purified into W state, which involves the one-photon-trapping phenomenon, with a high yield. Finally, an achievable setup for purification using only modest and presently feasible technologies is also proposed.
\end{abstract}

Quantum information and computation bring together the concepts from classical information science, computation theory, and quantum physics. The EPR-Bell[1] correlation, which occurs only when the quantum systems are entangled, generates the modern division between quantum and classical information theory. The regulation methods of quantum information processing such as quantum teleportation[2], quantum data compression[3, 4], and quantum cryptography [5] rely on the transmission of maximally entangled pairs over quantum channels between a sender and a receiver. As it is well known, the quantum channels are always noisy due to inevitable interactions with environments[6]. The pairs shared by the parties may become undesired mixed states. For this reason, great attentions have been focused on the agreement

${ }^{*}$ Corresponding author email address: dschuu@mail.nctu.edu.tw; Fax:886-3-5725230; Tel:886-3-571212156105 . 
of entanglement purification[7], schemes of entanglement distillation[8], and the decoherence mechanisms of quantum bits (qubits) in a reservoir[9].

Although the environment leads to the decoherence of the qubits, it may play an active role on the formation of the nonlocal effect under well considerations. Recently, many investigations[10] have been devoted to the considerations of the reservoir-induced entanglement between two remote qubits. By utilizing the the coupled field of bipartite systems[11, 12, 13] or, generally speaking, manipulating a third system which interacts with two remote qubits[14], many schemes have been proposed to enhance the entanglement fidelity. The scenario usually associates with a composite quantum system consisting two two-level subsystems inside a leaking optical cavity with the purpose of taking unintermitted monitor on the coupled field state. These proposals are only applicable in specific situations that the subsystems and the coupled field are in well prepared states, or in certain instant that the degree of entanglement is maximum. However, more general situations are still lacking in a entanglement distillation or entanglement generation process, especially the extension of the proposed two-particle schemes based on conditioned measurements to the protocol of multi-particle entanglement generation. This issue is crucial for both the real application of quantum communication[15] and the study on the mechanism of multi-particle entanglement.

In this paper, we present a notion of three-particle entanglement purification and an achievable setup using only modest and presently feasible technologies. The physical system undertaken for entanglement purification is dot-like single quantum well exciton coupled through a single microcavity mode. The proposed scheme is depicted in Fig.1. The whole procedure in the scheme is based on optical initialization, manipulation, and read-out of exciton state. In it, the qubit is coded in the presence of an exciton in a quantum well (QW), i.e. the exciton state in $i$ th $\mathrm{QW},|e, h\rangle_{i}$, is considered as the logical state $|0\rangle_{i}$, and the vacuum state, $|0,0\rangle_{i}$, which represents the state with no electron and hole, is coded as the logical state $|1\rangle_{i}$. The theoretical framework for the proposed scheme is based on the quantum jump approach[16]. To analyze the evolution of the double-exciton entanglement, a series of conditional measurement has been taken on the cavity field state. For accomplishing conditional measurements faithfully, the injection and leak of the cavity photon are controlled by means of the electro-optic effect. Therefore, the photon field plays an active role in the procedure of purification. According to our results of analysis, the proposed scheme can search for the decoherence-free state, i.e. the maximally entangled state, of the qubit-reservoir system in a time shorter than the characteristic time of the qubit system. Furthermore, a high purification yield and nearly unit of the entanglement fidelity can be acquired in our proposal.

First, we consider that our system consists of only two dot-like single QWs embedded inside a single-mode microcavity with the same coupling constant. We assume that the lateral size of the two QWs are sufficiently larger than the Bohr radius of excitons but smaller than the wavelength of the photon fields. Therefore, the dipole-dipole interactions and other nonlinear interactions can be neglected. The cavity mode is assumed to be resonant with the exitons. Under the rotating wave approximation, the unitary time evolution of the system is then governed by the interaction picture Hamiltonian 


$$
H_{2(I)}=\sum_{n=1}^{2} \hbar \gamma\left(a \sigma_{n}^{+}+a^{+} \sigma_{n}^{-}\right),
$$

where $\gamma$ is the coupling constant, $a^{+}(a)$ is the creation (annihilation) operator of the cavity field, and $\sigma_{n}^{+}\left(\sigma_{n}\right)$ represents the creation (annihilation) operator of the excitons in the $i$ th QW.

The dynamics of the double-dot excitons in the cavity consists of three processes: one- and two-photon absorption, one- and two-photon emission, and no photon absorption or emission. Since the formation of the maximally entangled exciton is a photon-trapping phenomenon, the main idea of the proposed scheme is to find out an eigenstate involved in the process of onephoton absorption and emission. Obviously, if the total number of quanta in the system is, $m$, there would be an eignstates of the Hamiltonian associates with the photon-trapping process, namely

$$
|\phi\rangle=\frac{1}{\sqrt{2}}\left(|1\rangle_{1} \otimes|0\rangle_{2}-|0\rangle_{1} \otimes|1\rangle_{2}\right) \otimes|m-1\rangle_{c},
$$

where $|m-1\rangle_{c}$ refers to the cavity field state with $m-1$ quanta. Once the system is in this state, the whole system does not decay at all. Accordingly, keeping the cavity mode in state $|m-1\rangle_{c}$, i.e. destroying the population of the cavity field state, paves the way to generate the entangled excitons

$$
|\psi\rangle=\frac{1}{\sqrt{2}}\left(|1\rangle_{1} \otimes|0\rangle_{2}-|0\rangle_{1} \otimes|1\rangle_{2}\right) .
$$

Therefore, taking a measurement on the cavity mode in order to see whether its state remains in the state or not is the key in our scheme.

For two-particle entanglement purification, the two dot-like single QWs and cavity mode is prepared in the vacuum state, $\left|\phi_{0}\right\rangle=|0\rangle_{1} \otimes|0\rangle_{2} \otimes|0\rangle_{c}$. A laser pulse, which is tuned to the lowest interband excitation energy, is then applied on the first QW. It can promote an electron from the valence-band to the conduction-band, hence the state, $\left|\phi_{0}\right\rangle=|1\rangle_{1}\left|0_{2}\right\rangle|0\rangle_{c}$, prepared for purification is obtained. For the sake of generality and purpose of distillation, the state of QW can be any mixed state, $\rho_{\psi}$, except the vacuum state. Next, a pulse with $m$ photons is injected into the microcavity. For the feasibility and the modest technology requirements, we consider here the injection of single photon, i.e. $m=1$. The total number of quantum count of the system is two.

As the single-photon has been injected into the cavity, the total system will evolve with time, and is governed by the operator

$$
U(t)=\left(\begin{array}{cccc}
2 \gamma^{2} a(G-K) a^{+}+1 & -i \gamma a S & -i \gamma a S & 2 \gamma^{2} a(G-K) a \\
-i \gamma S a^{+} & \frac{1}{2}(\cos (\mu t)+1) & \frac{1}{2}(\cos (\mu t)-1) & -i \gamma S a \\
-i \gamma S a^{+} & \frac{1}{2}(\cos (\mu t)-1) & \frac{1}{2}(\cos (\mu t)+1) & -i \gamma S a \\
2 \gamma^{2} a^{+}(G-K) a^{+} & -i \gamma a^{+} S & -i \gamma a^{+} S & 2 \gamma^{2} a^{+}(G-K) a+1
\end{array}\right)
$$

where $\mu^{2}=K^{-1}=2 \gamma^{2}\left(2 a^{+} a+1\right), G=K \cos (\mu t)$, and $S=\mu^{-1} \sin (\mu t)$. If the system evolves without interruption, it will go into a QW1- QW2-cavity field entangled state. If we take a 
measurement on the cavity field state at some instant, the number of the photon count of the detector may be one, two, or zero. Since the single-photon state $|1\rangle_{c}$ involves the photontrapping phenomenon, we can infer that the double-QW will evolve into a maximal entangled state if the cavity mode stays in state $|1\rangle_{c}$ via the quantum jump approach[16]. After measuring the cavity field state, injecting a subsequent photon is necessary for the sake of keeping the photon in its state. We then let the whole system evolve for another period of time $\tau$. Again, we proceed to measure the cavity photon to make sure whether its quantum is one or not. If the cavity photon remains in single-photon, the repetition continues; if not, the whole procedure should be started over. Therefore, after several times of successful repetitions, the state of double-exciton progresses into the state

$$
\rho_{\psi_{N}}=\left({ }_{c}\langle 1|U(\tau)| 1\rangle_{c}\right)^{N} \rho_{\psi_{i}}\left({ }_{c}\langle 1|U(-\tau)| 1\rangle_{c}\right)^{N} / P_{N}
$$

where

$$
P_{N}=\operatorname{Tr}\left[\left({ }_{c}\langle 1|U(\tau)| 1\rangle_{c}\right)^{N} \rho_{\psi_{i}}\left({ }_{c}\langle 1|U(-\tau)| 1\rangle_{c}\right)^{N}\right]
$$

is the probability of success for measuring a single-photon after $N$ times of repetitions. The superoperator, ${ }_{c}\langle 1|U(\tau)| 1\rangle_{c}$, reveals the significant fact that it will evolve to the projection operator, $|\psi\rangle\langle\psi|$, as the successful repetitions increases. This result comes from the fact that the superoperator, ${ }_{c}\langle 1|U(\tau)| 1\rangle_{c}$, has only one eigenvalue which its absolute value equals to one[14], here, the corresponding eigenvector is just the photon-trapping state. Thus the double-exciton state will become a maximal entangled state as long as the repetitions is sufficient large. By Eq. (6), the probability of success can be evaluated

$$
P_{N, 1}=\frac{1}{2}\left(1+\cos (\sqrt{6} \gamma \tau)^{2 N}\right)
$$

and the fidelity is

$$
\begin{aligned}
F_{N, 1} & =\left\langle\psi\left|\rho_{\psi_{N}}\right| \psi\right\rangle \\
& =\frac{1}{2 \cos (\sqrt{6} \gamma \tau)^{2 N}}
\end{aligned}
$$

Here, if the product, $\gamma \tau$, is set to be $n \pi / \sqrt{6}, n=1,2, \ldots$, then the fidelity of the double-exciton state will approach to one and the probability of success will be $1 / 2$ as $N$ increases.

If the state of the cavity field is kept in $n$-photon state, rather than the state with single photon, Eq. (7) and Eq. (8) can be generalized to

$$
P_{N, n}=\frac{1}{2}\left(1+\cos (\sqrt{2(n+1)} \gamma \tau)^{2 N}\right)
$$

and

$$
F_{N, n}=\frac{1}{2 \cos (\sqrt{2(n+1)} \gamma \tau)^{2 N}}
$$


It reveals that the number of measured cavity photon and evolution period play important roles in the trade-off between $P_{N, n}$ and $F_{N, n}$. We can choose a set of $(n, \gamma \tau)$ such that the fidelity progresses to one at the least repetitions, however, in the same time it causes the probability to reduce to a minimum. This is the usual case for purification. On the other hand, one can also find a suitable such that the probability goes to one. In this case, the system will not evolve with time and is similar to the Zeno paradox with finite duration between two measurements.

The concept of two-particle entanglement purification discussed above can be generalized to three-particle case directly. Here, the same approximations have been made as in the doubleexciton case, and the formulation of three-exciton dynamics can be derived straightforwardly via the interaction Hamiltonian

$$
H_{3(I)}=\hbar \gamma\left(\begin{array}{cccccccc}
0 & a & a & 0 & a & 0 & 0 & 0 \\
a^{+} & 0 & 0 & a & 0 & a & 0 & 0 \\
a^{+} & 0 & 0 & a & 0 & 0 & a & 0 \\
0 & a^{+} & a^{+} & 0 & 0 & 0 & 0 & a \\
a^{+} & 0 & 0 & 0 & 0 & a & a & 0 \\
0 & a^{+} & 0 & 0 & a^{+} & 0 & 0 & a \\
0 & 0 & a^{+} & 0 & a^{+} & 0 & 0 & a \\
0 & 0 & 0 & a^{+} & 0 & a^{+} & a^{+} & 0
\end{array}\right) .
$$

The superoperator that governs the progress of the three dot-like single QWs with single-photon can be worked out:

$$
\begin{aligned}
{ }_{c}\left\langle 1\left|e^{i H_{3(I)} \tau}\right| 1\right\rangle_{c}= & g|g\rangle\left\langle g\left|+W_{1}\right| W_{1}\right\rangle\left\langle W_{1}\left|+T_{1}\right| T_{1}\right\rangle\left\langle T_{1}\left|++T_{2}\right| T_{2}\right\rangle\left\langle T_{2}\right| \\
& +e|e\rangle\left\langle e\left|+W_{2}\right| W_{2}\right\rangle\left\langle W_{2}\left|+T_{3}\right| T_{3}\right\rangle\left\langle T_{3}\left|++T_{4}\right| T_{4}\right\rangle\left\langle T_{4}\right|,
\end{aligned}
$$

where $g, e, W_{1}, W_{2}, T_{1}, T_{2}, T_{3}$ and $T_{4}$ are functions of $\tau$ which correspond to the orthonormal eigenvectors

$$
\begin{aligned}
|g\rangle & =|0\rangle_{1} \otimes|0\rangle_{2} \otimes|0\rangle_{3}, \\
\left|W_{1}\right\rangle & =\frac{1}{\sqrt{3}}\left(|1\rangle_{1} \otimes|0\rangle_{2} \otimes|0\rangle_{3}+|0\rangle_{1} \otimes|1\rangle_{2} \otimes|0\rangle_{3}+|0\rangle_{1} \otimes|0\rangle_{2},\right. \\
\left|T_{1}\right\rangle & =\frac{1}{\sqrt{2}}\left(|1\rangle_{1} \otimes|0\rangle_{2} \otimes|0\rangle_{3}-|0\rangle_{1} \otimes|0\rangle_{2} \otimes|1\rangle_{3}\right), \\
\left|T_{2}\right\rangle & =\frac{1}{\sqrt{6}}\left(|1\rangle_{1} \otimes|0\rangle_{2} \otimes|0\rangle_{3}-2|1\rangle_{1} \otimes|0\rangle_{2} \otimes|1\rangle_{3}+|0\rangle_{1} \otimes|0\rangle_{2} \otimes|1\rangle_{3}\right), \\
|e\rangle & =|1\rangle_{1} \otimes|1\rangle_{2} \otimes|1\rangle_{3}, \\
\left|W_{2}\right\rangle & =\frac{1}{\sqrt{3}}\left(|0\rangle_{1} \otimes|1\rangle_{2} \otimes|1\rangle_{3}+|1\rangle_{1} \otimes|0\rangle_{2} \otimes|1\rangle_{3}+|1\rangle_{1} \otimes|1\rangle_{2} \otimes|0\rangle_{3}\right), \\
\left|T_{3}\right\rangle & =\frac{1}{\sqrt{2}}\left(|0\rangle_{1} \otimes|1\rangle_{2} \otimes|1\rangle_{3}-|1\rangle_{1} \otimes|1\rangle_{2} \otimes|0\rangle_{3}\right), \\
\text { and }\left|T_{4}\right\rangle & =\frac{1}{\sqrt{6}}\left(|0\rangle_{1} \otimes|1\rangle_{2} \otimes|1\rangle_{3}-2|0\rangle_{1} \otimes|1\rangle_{2} \otimes|0\rangle_{3}+|1\rangle_{1} \otimes|1\rangle_{2} \otimes|0\rangle_{3}\right) .
\end{aligned}
$$


Here $T_{1}=T_{2}$ and $T_{3}=T_{4}$ are two two-fold degenerate eigenvalues of the superoperator ${ }_{c}\left\langle 1\left|e^{i H_{3(I)} \tau}\right| 1\right\rangle_{c}$.

If the initial state is set equal to

$$
\left|\phi_{i}\right\rangle=|1\rangle_{1} \otimes|0\rangle_{2} \otimes|0\rangle_{3} \otimes|0\rangle_{c}
$$

, the probability of success for finding the exciton state is in state $\left|W_{1}\right\rangle$ can then be worked out analytically:

$$
P_{N}=\frac{1}{3}\left(\cos (\sqrt{10} \gamma \tau)^{2 N}+2 \cos (\gamma \tau)^{2 N}\right),
$$

and the corresponding fidelity of the three-exciton state is

$$
\begin{aligned}
F_{N} & =\left\langle W_{1}\left|\rho_{\psi_{N}}\right| W_{1}\right\rangle \\
& =\frac{\cos (\sqrt{10} \gamma \tau)^{2 N}}{\cos (\sqrt{10} \gamma \tau)^{2 N}+2 \cos (\gamma \tau)^{2 N}}
\end{aligned}
$$

Here, we can set $\gamma \tau$ to be $n \pi / \sqrt{10}, n=1,2, \ldots$, then the fidelity of the three-exciton state approaches to unit as $N$ increases; meanwhile the probability of success is $1 / 3$. A special case, $\gamma \tau=\pi / \sqrt{6}$, is demonstrated in Fig. 2. Fig. 2(a) describes the trade-off between $P_{N}$ and $F_{N}$. The purification yield $Y_{N}=\prod_{i=0}^{N} P_{i}$, which measures the fraction of surviving pairs, is also presented in Fig. 2(b).

Greenberger-Horn-Zeilinger (GHZ)[17] and W states[18, 19] are two kinds of three-particle maximally entanglement. The former involves three-photon trapping phenomenon which can be described through the state vector

$$
\left|\psi_{\mathrm{GHZ}}\right\rangle=\frac{1}{\sqrt{3}}\left(|0\rangle_{1} \otimes|0\rangle_{2} \otimes|0\rangle_{3}-|1\rangle_{1} \otimes|1\rangle_{2} \otimes|1\rangle_{3}\right) .
$$

and $\mathrm{W}$ state is a one-photon trapping state

$$
\left|\psi_{\mathrm{W}}\right\rangle=\frac{1}{\sqrt{3}}\left(|1\rangle_{1} \otimes|0\rangle_{2} \otimes|0\rangle_{3}+|0\rangle_{1} \otimes|1\rangle_{2} \otimes|0\rangle_{3}+|0\rangle_{1} \otimes|0\rangle_{2} \otimes|1\rangle_{3}\right)
$$

Although these states associated with different physical phenomenon, they possess the same degree of entanglement $[18,19]$. In our scheme, any initial mixed state of the three-exciton state can be purified into $\mathrm{W}$ state except the vacuum. However, the symmetry properties of qubitenvironment interactions for GHZ state is quite different from three-exciton W state except the vacuum, thus it can not be generated via the approach of conditional measurement.

The whole concepts for the experiments are schematically shown in Fig. 3. A Ti:sapphire laser supplies the pulse light source for all of the operation photons in our devices such as resonant photon, $2 \omega$ photon, and pulse $\mathbf{E}$ field. The timing between all of the operation photons could be controlled precisely by delay stages. Also, the detection of photodiodes could be precisely triggered by laser pulses. Firstly, one of the dot-like single quantum wells is excited by the $3 \mathrm{eV}$ photon from $2 \omega$ generator performed through a nonlinear crystal, e.g. BBO or LBO crystal. Then, a resonant photon with vertical linear polarization generated via a quartz plate 
is injected into the cavity which is constructed by the ZnTe with both Au films. Meanwhile, through the pulse $\mathbf{E}$ field[15] with appropriate magnitude[18] and pulse width of $3.3 p s$ as shown in Fig. 4, the linear polarization of injected photon is rotated from vertical to horizontal via the Electro-optic effect in $\mathrm{ZnTe}[19]$. After the sufficient evolution time $\left(T_{e v} \sim 20 p s\right.$, the time period, $\tau$, between two measurements) with dot-like single quantum well excitons, the photon in cavity can be leaked out the cavity by a pulse $\mathbf{E}$ field with suitable timing and detected by a single photon avalanche diode (SPAD, detector 2 in Fig. 3). This procedure would be repeated until finishing the purification. During this procedure, the photoluminescence lifetime of dot-like single quantum well exciton can be measured by the other SPAD as shown in Fig. 4 (detector 1 in Fig. 3).

To summarize, a three-particle entanglement purification scheme based on conditioned measurements has been proposed in this work. We investigate the entanglement generation of dot-like single quantum well excitons coupled through a single microcavity mode. As shown in Eq. (9) and Eq. (10), we first consider how the cavity photon affects the purity of the exciton state and the purification efficiency in two-particle protocol. The trade-off between $P_{N, n}$ and $F_{N, n}$ reveals whether the double excitons can be purified into a photon trapping, decoherence-free state efficiently depending on the number of photon counts in the repeated measurements. Moreover, we conclude that the three-exciton state can be purified into $\mathrm{W}$ state with a well yield but smaller than that in two-particle case. Finally, a feasible experimental setup for optical initialization, manipulation, and read-out of exciton state is also presented.

This work is supported partially by the National Science Council, Taiwan under the grant number NSC 92-2120-M-009-010.

\section{Figure Caption}

Fig. 1. The quantum devices with triple dot-like quantum wells inlaid in a microcavity which is constructed by a ZnTe medium and two Au mirrors, will be prepared by the MBE, the e-beam lithography, and the conventional semiconductor processing.

Fig. 2. (a) The probability of success for measuring the same photon state, $P_{N}$, and the fidelity of the exciton state, $F_{N}$, and (b) the corresponding yield, $Y_{N}$, for $\mathrm{W}$ state generation.

Fig. 3. The whole experimental concepts for optical initialization, manipulation, and read-out of exciton state.

Fig. 4. The flowchart for $\mathrm{W}$ state generation. The inset quantum device is the same with the device in Fig. 1.

\section{References}

[1] A. Einstein, B. Podolsky, and N. Rosen, Phys. Rev. 47, 777 (1935); J. S. Bell, Rev. Mod. Phys. 38, 447 (1966).

[2] C. H. Bennett, G. Brassard, C. Crepeau, R. Jozsa, A. Peres, and W. K. Wootters, Phys. Rev. Lett., 70, 1895 (1993). 
[3] B. Schumacher, Phys. Rev. A, 51, 2738 (1995).

[4] R. Jozsa and B. Schumacher, J. Modern Optics, 41, 2343 (1994).

[5] A. K. Ekert, Phys. Rev. Lett., 67, 661 (1991).

[6] W. H. Zuerk, Mod. Phys. 75, 715 (2003).

[7] C. H. Bennett, G. Brassard, S. Popescu, B. Schumacher, J. A. Smolin, and W. K. Wootters, Phys. Rev. Lett., 76, 722 (1996); D. Deutsch, A. Ekert, R. Jozsa, C. Macchiavello, S. Popescu, and A. Sanpera, Phys. Rev. Lett., 77, 2818 (1996); J.-Y. Hsieh, C.-M. Li, and D.-S. Chuu, Physics Letters A, 328, 94 (2004).

[8] L.-M. Duan, M. D. Lukin, J. I. Cirac, and P. Zoller, Science, 414, 413 (2001).

[9] S. Bose, I. Fuentes-Guridi, P. L. Knight, and V. Vedral, Phys. Rev. Lett. 87, 050401 (2001); 87, 279901(E) (2001); R. W. Rendell and A. K. Rajagopal, Phys. Rev. A 67, 062110 (2003).

[10] Daniel Braun, Phys. Rev. Lett. 89, 277901 (2002); Y. N. Chen, D. S. Chuu, and T. Brandes, Phys. Rev. Lett. 90, 166802 (2003) ; M. Paternostro, W. Son, and M. S. Kim, Phys. Rev. Lett. 92, 197901 (2004).

[11] X. X. Yi, C. S. Yu, L. Zhou, and H. S. Song, Phys. Rev. A 68, 052304 (2003).

[12] C. Cabrillo, J.I. Cirac, P. Garcia-Fernandez, and P. Zoller, Phys. Rev. A 59, 1025 (1999)

[13] M. Plenio, S.F. Huelga, A. Beige, and P.L. Knight, Phys. Rev. A 59, 2468 (1999).

[14] H. Nakazato, T. Takazawa, and K. Yuasa, Phys. Rev. Lett. 90, 060401 (2003); H. Nakazato, M. Unoki, and K. Yuasa, Phys. Rev. A 70, 012303 (2004); G. Compagno, A. Messina, H. Nakazato, A. Napoli, M. Unoki, and K. Yuasa, Report No. quant-ph/0405074.; L.-A. Wu, D. A. Lidar, S. Schneider, Phys. Rev. A 70, 032322 (2004).

[15] J. Joo, Y. Park, S. Oh and J. Kim, New Journal of Physics 5, 136 (2003).

[16] M. Plenio and P.L. Knight,, Rev. Mod. Phys. 70, 101 (1998).

[17] D. M. Greenberger, M. A. Horne, and A. Zeilinger, in Bell's Theorem, Quantum Theory, and Conceptions of the Universe, edited by M. Kafatos (Kluwer, Dordrecht, 1989), p. 69.

[18] W. Dur, G. Vidal, and J. I. Cirac, Phys. Rev. A 62, 062314 (2000).

[19] A. Acin, D. Brub, M. Lewenstein, and A. Sanpera, Phys. Rev. Lett. 87, 040401 (2001).

[20] Actually, the pulse E field is the "THz radiation" which could be easily generated from GaAs semiconductor or several nonlinear crystal such as GaSe [21, 22].

[21] K. Reimann, et al., Opt. Lett. 28, 471 (2003). 
[22] C. W. Luo, et al., Semicond. Sci. Technol. 19, S285 (2004).

[23] According to the electro-optic modulation theory, the half-wave voltage derived from phase retardation between principal axes is given by $V \pi=(d \lambda) /\left(L 2 n^{3} r_{41}\right)$. For our device, where $d=2 \mu \mathrm{m}$ is the thickness of ZnTe and is along the pulse $\mathbf{E}$ field, $L=1 \mathrm{~mm}$ is the length of ZnTe, i.e. the optical path length of injected photon, $\lambda=713 \mathrm{~nm}$ is the wavelength of injected photon, $n=2.906$ is the index of refraction for $713 \mathrm{~nm}, r_{41}=3.9^{-12} \mathrm{~m} / \mathrm{V}$ is the electro-optic coefficient. Then we can evaluate that $V \pi=7.45 \mathrm{~V}$. We need $\sim .45 \mathrm{~V}$ to rotate the polarization of injected photon from vertical to horizontal. This means that the magnitude of pulse E field with the ZnTe of $2 \mu \mathrm{m}$ thickness is $\sim 37.25 \mathrm{KV} / \mathrm{cm}$.

[24] Q. Wu and X.-C. Zhang, Appl. Phys. Lett. 71, 1285 (1997). 


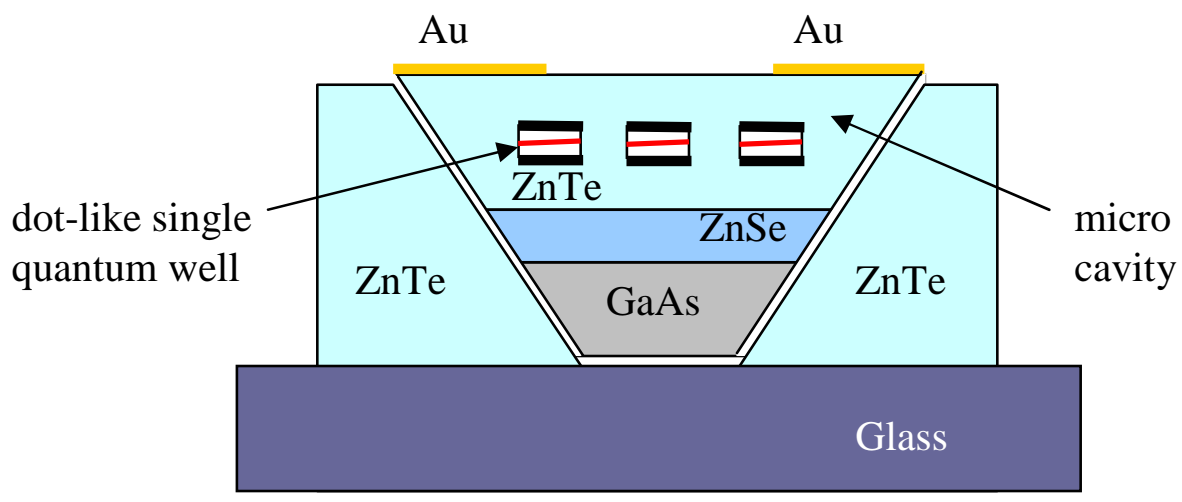

Fig. 1 


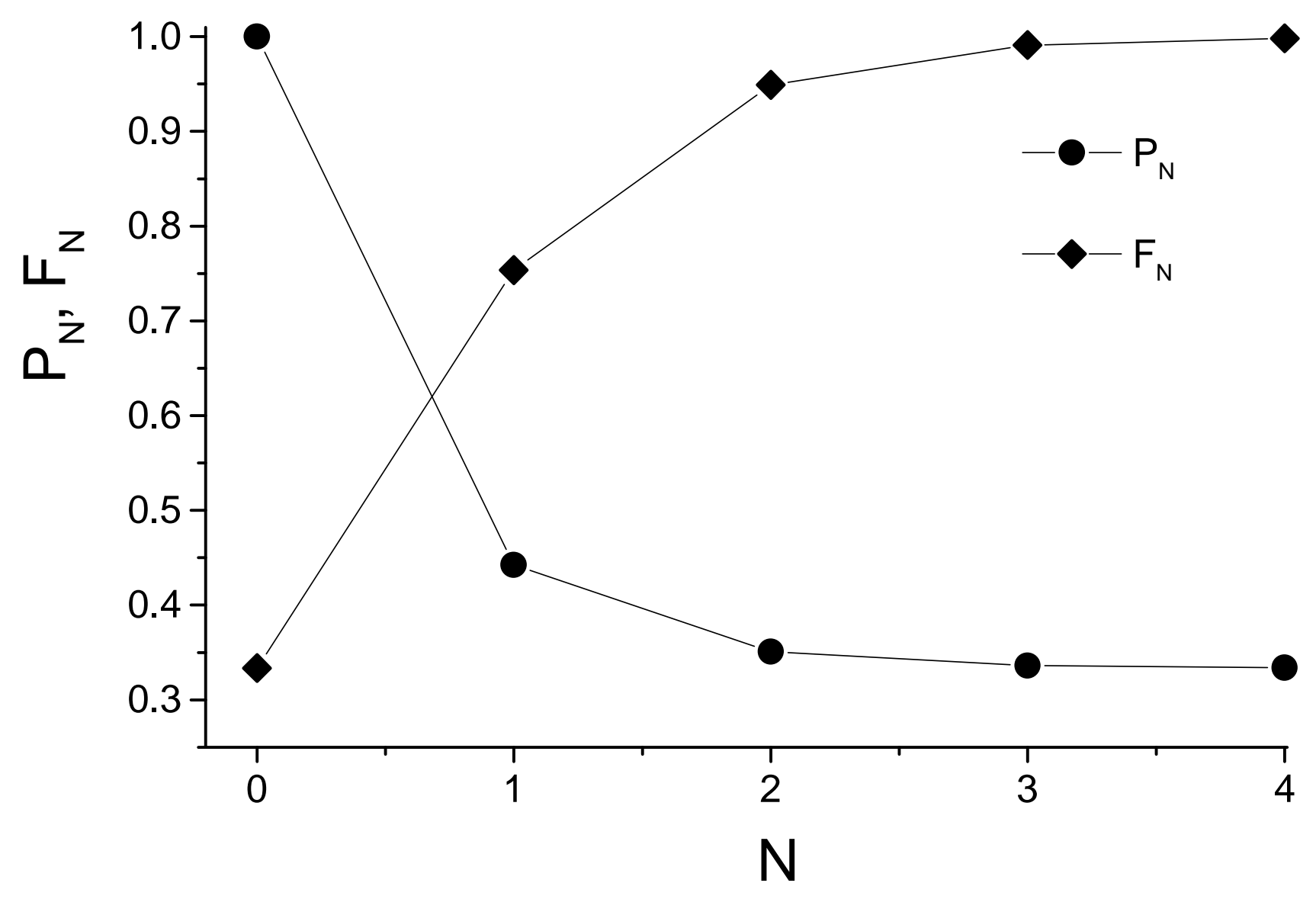

Fig. 2 (a) 


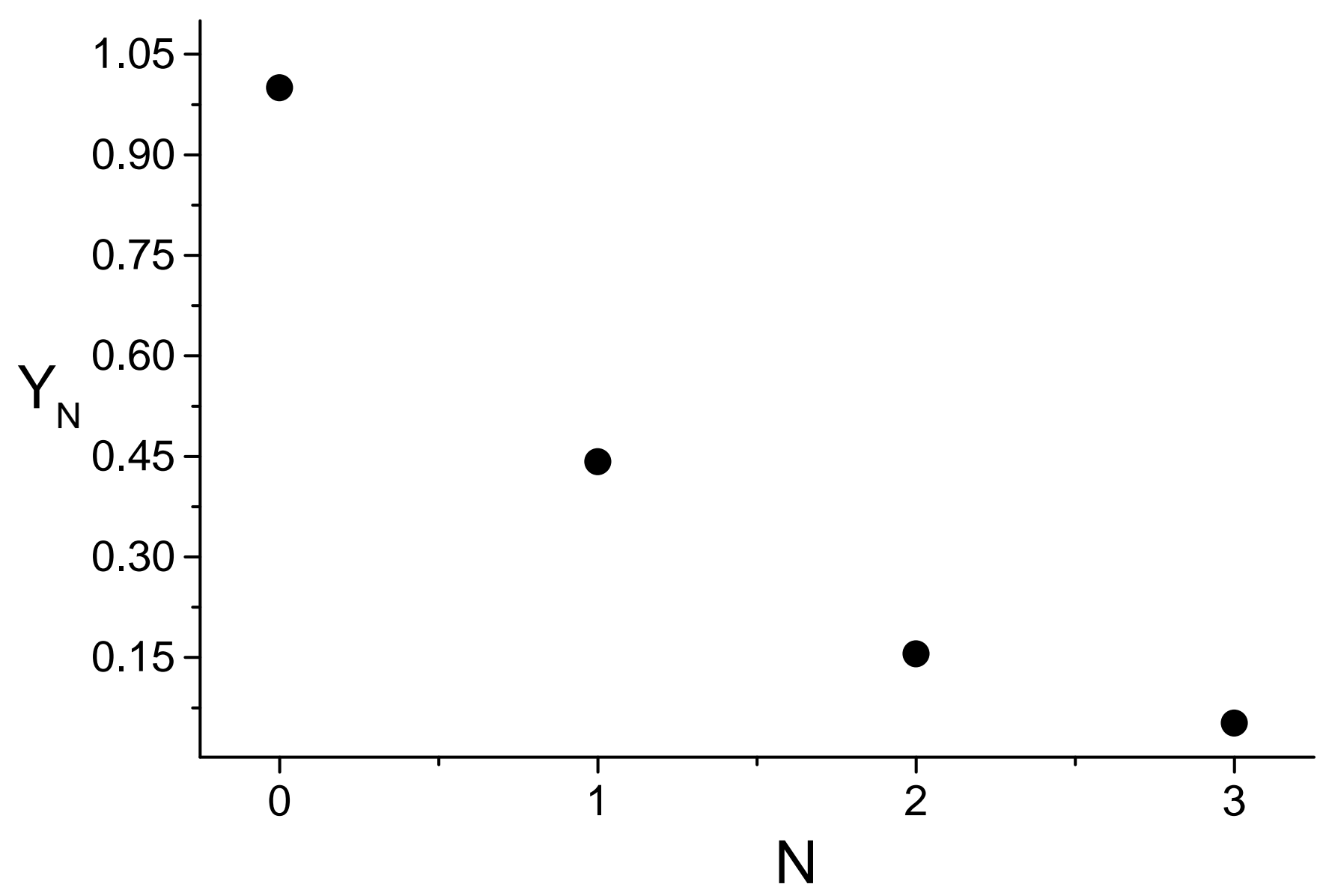

Fig. 2(b) 


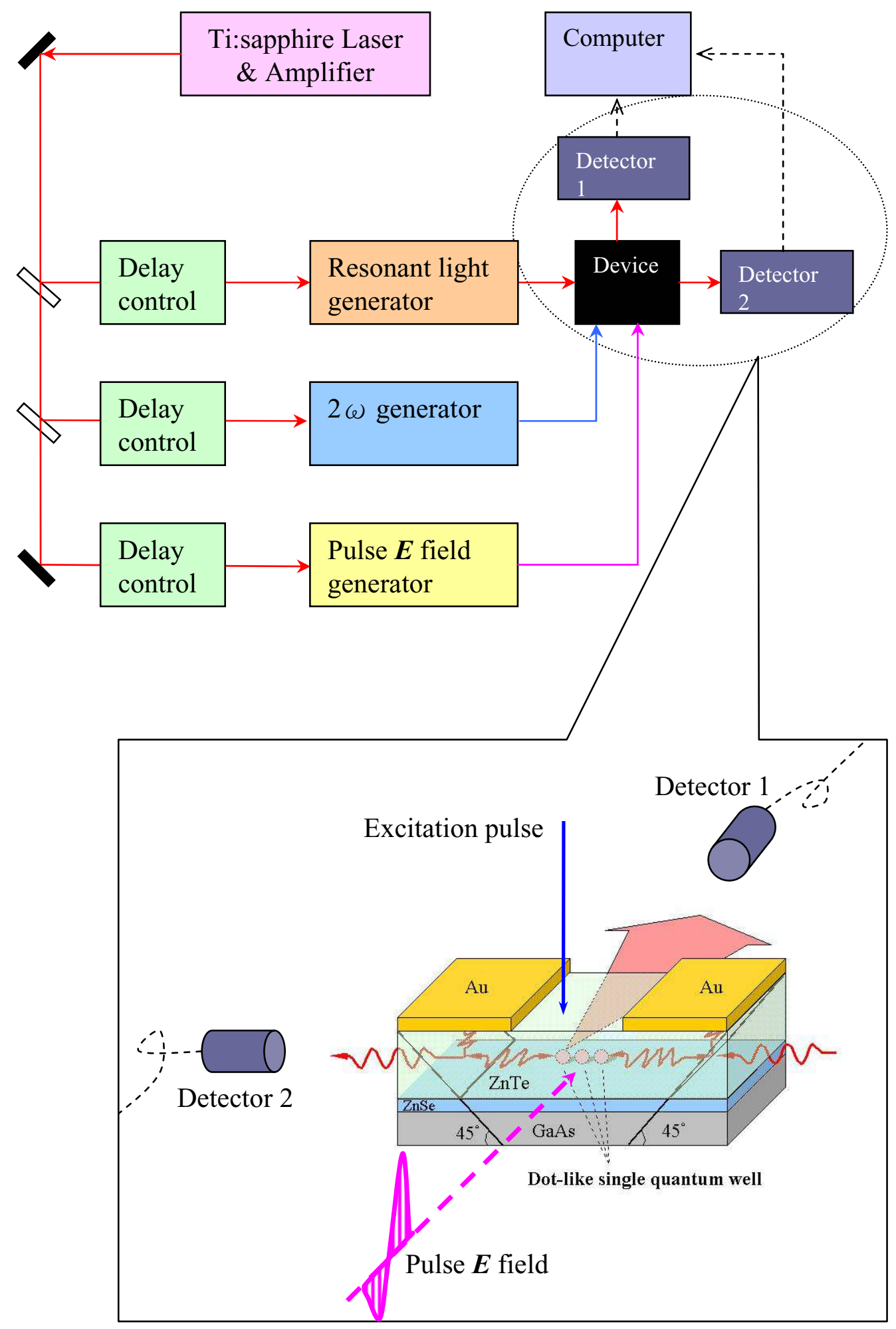

Fig. 3 


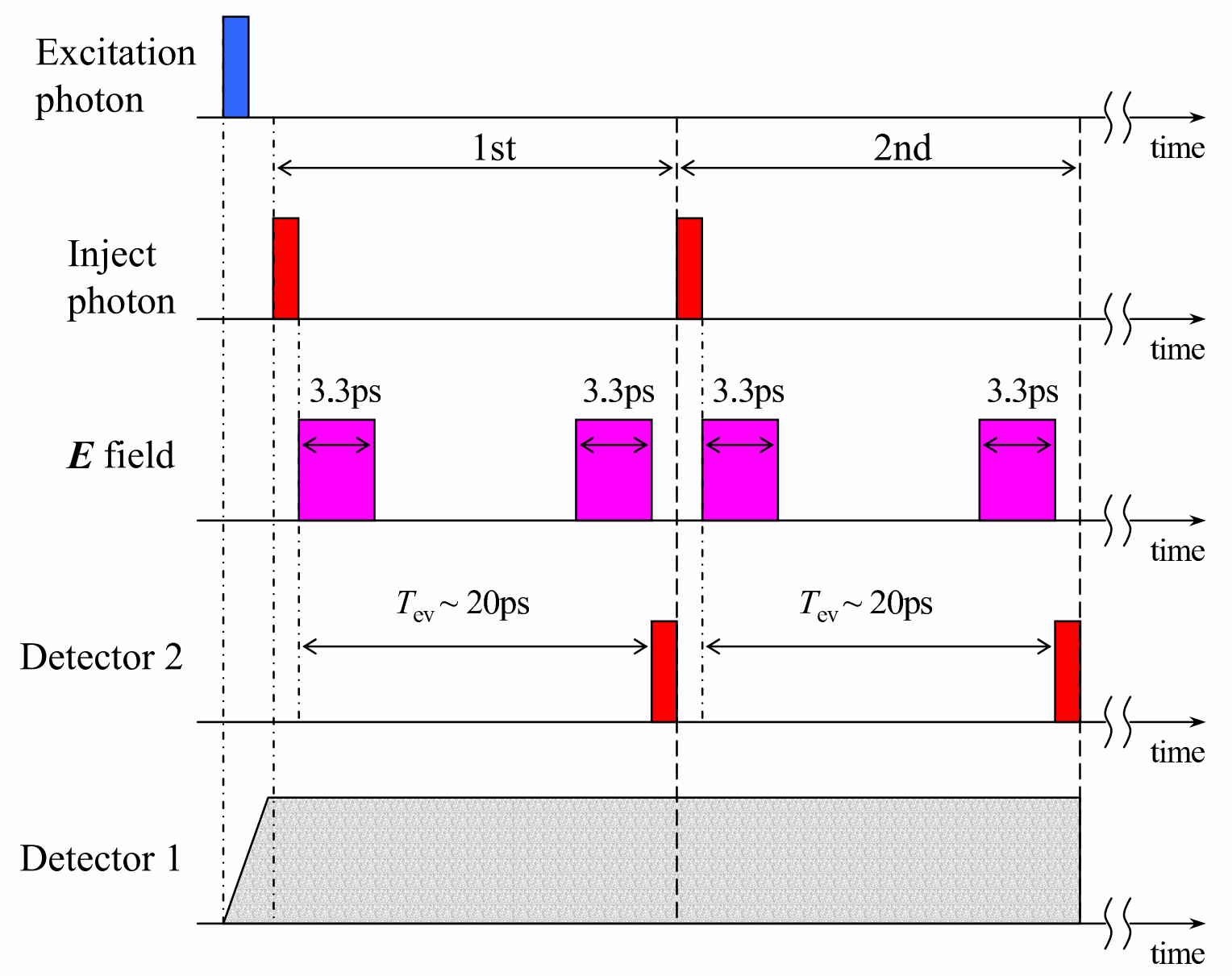

Fig. 4 